\title{
Nicho trófico de la nutria neotropical (Lontra longicaudis) en un ambiente modificado, Temascaltepec, México
}

\author{
Food niche of the neotropical river otter (Lontra longicaudis) in a modified environment, \\ Temascaltepec, México
}

\author{
Octavio Monroy-Vilchis* y Víctor Mundo \\ Estación Biológica Sierra Nanchititla, Facultad de Ciencias, Universidad Autónoma del Estado de México. Instituto Literario 100. Centro, 50000 \\ Toluca, Estado de México, México. \\ *Correspondencia: omv@uaemex.mx; tavomonroyvilchis@gmail.com
}

\begin{abstract}
Resumen. Se analizaron los cambios en espacio y tiempo de dieta de la nutria en Temascaltepec, Estado de México, en 2 ríos que presentan importante intervención humana causada por el establecimiento de criaderos de peces comerciales. Se recolectaron 157 excrementos, identificandose la trucha (Oncorhynchus mykiss) como la presa más consumida (92.4\%), seguida por invertebrados (3.5\%), anfibios $(2.9 \%)$ y plantas $(1.8 \%)$. La dieta de la nutria no varía significativamente entre estaciones climáticas, mientras que entre ríos, la variación fue significativa. En la zona de estudio, la nutria muestra tendencia a especializarse en el consumo de trucha. Las granjas de trucha influyen en la dieta de la nutria y se presentan como clave para la permanencia de la nutria en la zona de estudio.
\end{abstract}

Palabras clave: carnívoros, dieta, ecología, especialista, Estado de México, Oncorhynchus mykiss.

\begin{abstract}
Spatial and temporal changes in the diet of the river otter in Temascaltepec, Mexico were analyzed on 2 rivers that present important human disturbance caused by aquaculture of commercial fish. 157 scats were collected, the principal prey was trout Oncorhynchus mykiss (92.4\%) followed by invertebrates (3.5\%), amphibians (2.9\%) and plants $(1.8 \%)$. Between climatic stations the diet of the river otter did not vary significantly, whereas between rivers it presented significant variation. The river otter showed specialization in the consumption of trout. The trout farms have influenced the diet of the river otter, and appear as key for the permanence of the otter in the study zone.
\end{abstract}

Key words: carnivores, diet, ecology, specialist, State of Mexico, Oncorhynchus mykiss.

\section{Introducción}

Los estudios de dieta son importantes en la ecología de los vertebrados, en particular de mamíferos carnívoros (Gittleman, 1989; Monroy-Vilchis et al., 2009). En los carnívoros, el alimento puede condicionar la organización social, el uso de hábitat y las tasas de reproducción (Braña et al., 1987). La dieta de carnívoros es un aspecto ampliamente estudiado, cuyo enfoque tradicional ha sido entender las interacciones que tienen con su medio y en particular con las especies presa (Servín y Huxley, 1991; Guerrero et al., 2002; Casariego-Madorell et al., 2006; Monroy-Vilchis et al., 2009), aunque recientemente el interés se ha dirigido a conocer el efecto de la depredación de estos organismos sobre otras especies de interés económico para el hombre,

Recibido: 23 octubre 2008; aceptado: 02 marzo 2009 como ganado, peces y crustáceos, entre otros (Michalski et al., 2006; Cascelli y Murray, 2007).

En la NOM-ECOL-59-2001(SEMARNAT, 2002), y en el Apéndice I de CITES (UNEP-WCMC, 2007), la nutria neotropical (Lontra longicaudis) se considera una especie amenazada en México; además, está protegida en Argentina, Bolivia, Brasil, Colombia, Costa Rica, Ecuador, Nicaragua, Panamá, Paraguay, Perú, Suriname, Trinidad, Tobago, Uruguay y Venezuela (Larivière, 1999). Lontra longicaudis, como cualquier otra especie, es sensible a cambios drásticos en su hábitat, a la degradación de los ambientes de ribera y a la contaminación de los cuerpos de agua, por lo que se le consideró un indicador de la degradación de los ecosistemas acuáticos (Lodé, 1993). Sin embargo, estudios recientes han revelado la presencia de la nutria en tramos de ríos encauzados y canales de riego con perturbaciones significativas y disminución 
de las especies que son sus presas principales (GalloReynoso, 1989; Larivière, 1999; Sierra-Huelsz y VargasContreras, 2002), evidenciando que existe cierta tolerancia a las alteraciones del hábitat que se consideraban como insuperables y que daban lugar a factores limitantes, lo que sugiere que la nutria puede sobrevivir aún en zonas con condiciones calificadas como extremas para su permanencia (Gallo-Reynoso, 1989; Kruuk, 1995; RuizOlmo y Delibes, 1998). Lo anterior muestra un aspecto interesante; conocer qué modificaciones o adaptaciones en los hábitos alimentarios han presentado las nutrias para poder mantenerse en este tipo de ambientes. A pesar de esto sus poblaciones se han visto fuertemente disminuidas en diferentes regiones debido a las condiciones generadas por la pérdida de hábitat, por contaminación del agua y destrucción de los ambientes de ribera, además de la cacería ilegal (Gallo-Reynoso, 1989; Soler 2002).

Los componentes de la dieta de la nutria son muy diversos; se mencionan crustáceos (Spínola y Vaughan, 1995), peces (Kasper et al., 2004) y aves (Gallo-Reynoso et al., 2008). En el norte de México los peces son el grupo más frecuente (Gallo-Reynoso, 1996) y en otras localidades del país, los crustáceos (Gallo-Reynoso, 1997); también en el sur de México los peces son el componente más abundantes en la dieta (Macías-Sánchez y Aranda, 1999; Ramón 2000). Estos estudios se han realizado en sitios relativamente bien conservados, por lo que es necesario aún conocer cómo es la ecología alimentaria de esta especie en sitios con perturbación considerable y de esta manera comprender las estrategias alimentarias que emplea la nutria para poder sobrevivir.

El objetivo del presente estudio es describir el nicho trófico del L. longicaudis en 2 ríos que reciben descargas directas urbanas e industriales, donde están presentes criaderos de trucha arco iris y comparar este nicho trófico con los obtenidos a partir de otros estudios.

Temascaltepec se encuentra al suroeste del Estado de México entre los $19^{\circ} 03^{\prime} \mathrm{N}$ y $100^{\circ} 02^{\prime} \mathrm{O}$, dentro de la faja volcánica transmexicana (Fig. 1). El clima es semicálido subhúmedo $\left(\mathrm{ACw}_{2}\right)$ con lluvias en verano (INEGI, 2008). La temperatura media anual oscila entre $\operatorname{los} 18^{\circ}$ y $22^{\circ} \mathrm{C}$. La precipitación anual varía de 800 a 1600 mm (Borboa, 1999). La vegetación está formada por bosque de encinopino, pastizal introducido, agricultura de temporal y de riego (INEGI, 2008). Los 2 ríos se localizan a menos de $15 \mathrm{~km}$ de la cabecera municipal y vierten sus aguas hacía la cuenca del Balsas, que cuenta con 30336 habitantes (INEGI, 2008), los desechos urbanos son vertidos directamente en los ríos, sobre éstos se establecen aproximadamente 10 criaderos de trucha localizados antes de las descargas urbanas (obs. per). Ambos ríos presentan cauces de aguas poco profundas que aumentan en la temporada de lluvias, el terreno en las

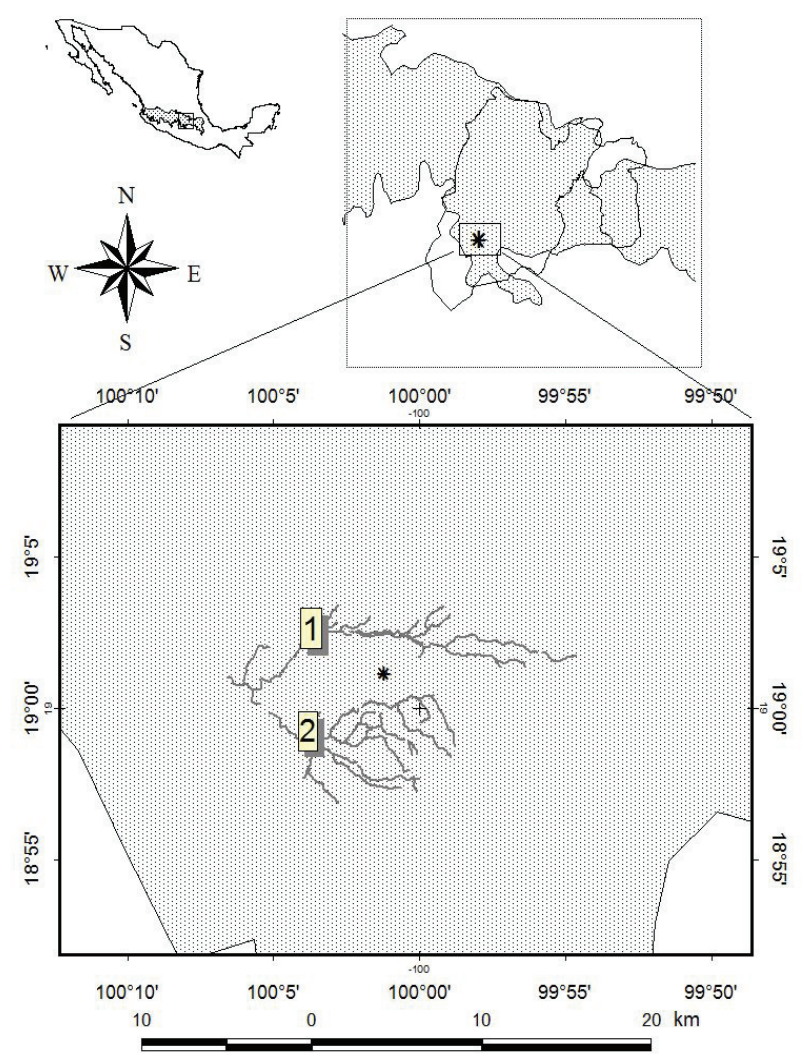

Figura 1. Ubicación de la zona de estudio dentro de la cuenca del río Balsas, mostrando el río Grande (1) y el río Telpintla (2).

orillas es variable, presentando zonas planas y acantilados y zonas que colindan con asentamientos humanos. Uno de los ríos (Telpintla) se localiza a una altitud de $1880 \mathrm{~m}$, con temperatura promedio de $15.7^{\circ} \mathrm{C}$, la concentración de oxígeno es de $8.5 \mathrm{mg} / \mathrm{l}$, los sólidos totales disueltos 54.86 $\mathrm{mg} / \mathrm{l}$, conductividad $115.5 \mathrm{~S} / \mathrm{m}$ y un $\mathrm{pH}$ de 6.25 . El otro río (Grande) está a $1700 \mathrm{~m}$ de altitud, con temperatura promedio de $15.3{ }^{\circ} \mathrm{C}$, concentración de oxígeno de 9.08 $\mathrm{mg} / \mathrm{l}$, sólidos totales disueltos $45.93 \mathrm{mg} / \mathrm{l}$, conductividad 103.4 S/m y pH de 6 (Guerrero, 2007).

\section{Materiales y métodos}

Mensualmente se recorrieron $6 \mathrm{~km}$ en cada río de noviembre de 2004 a junio de 2006; se recolectaron muestras de excrementos durante las 2 temporadas climáticas: seca (diciembre-mayo) y húmeda (junio-noviembre), con base en los criterios establecidos por Aranda (2000); se colocaron en bolsas de plástico, rotuladas con la fecha y posición geográfica. Las muestras se procesaron 
siguiendo el método propuesto por Gallo-Reynoso (1987) y modificado por Macías-Sánchez (1998) y usando como referencia la guía de pelos de Monroy-Vilchis y RubioRodríguez (2003). Los excrementos se colocaron en tamices (medias de nylon) que se sumergieron en un recipiente que contenía detergente comercial y se dejaron reposar durante una hora, después se lavaron con agua corriente y se secaron a temperatura ambiente. Posteriormente, se separaron los restos sólidos y se analizaron con ayuda de un estereoscopio. El reconocimiento de peces y anfibios se realizó mediante la recolecta de ejemplares en campo usando la identificación de vértebras, espinas y escamas, en el caso de peces, y de huesos femorales y escapulares, en el caso de anfibios. Para insectos se usaron guías taxonómicas de identificación (Fierros-López, 2003). Para la identificación de las plantas se recurrió a la ayuda de un especialista. El material se identificó hasta el nivel taxonómico posible.

Los resultados obtenidos se expresaron como frecuencia de presencia y porcentaje de aparición (Macías-Sánchez, 1998). Para determinar el tamaño de muestra necesario para describir la dieta de la nutria, se obtuvo el número mínimo de excrementos, calculando el porcentaje de presencia de las presas en 10 excrementos seleccionados al azar, y repitiendo esto, adicionando cada vez 5 muestras seleccionadas también al azar, hasta completar el total de muestras (Monroy-Vilchis et al., 2009). Las listas parciales de las frecuencias de las distintas presas ( $\mathrm{n}=10$ excrementos, $15,20,25, \mathrm{n} .$. ) se compararon con el listado total $(n=157)$ por medio de una prueba de $\mathrm{X}^{2}$ (Zar, 1999). El número de muestras en el cual no se presentaron diferencias estadísticamente significativas fue considerado como el tamaño mínimo de muestra necesario para describir la dieta de la nutria en la zona de estudio (Monroy-Vilchis et al., 2009). La variación temporal de la dieta entre ríos y entre temporadas se analizó utilizando una prueba de $X^{2}$ (Zar, 1999). Para conocer la amplitud del nicho trófico estandarizado se utilizó el índice de Levin (Krebs, 1999). Se revisaron los trabajos de dieta de L. longicaudis para otras zonas y se usaron aquellos que presentaban la información suficiente para obtener el nicho trófico estandarizado y se compararon entre sí y con este estudio. El solapamiento del nicho trófico se evaluó con el índice de Pianka (Krebs, 1999).

\section{Resultados}

Se colectó un total de 157 excrementos, en los que se identificaron 4 grupos de presas: peces, anfibios, insectos y plantas. El grupo mejor representado fue el de peces con $91.8 \%$ de aparición, seguido de insectos 3.5\%, anfibios
$2.9 \%$ y plantas $1.8 \%$ (Cuadro 1). Sólo se registró 1 especie de pez, la trucha arco iris (Oncorhynchus mykiss), 2 especies de anfibios, 1 rana y 1 sapo (Hyla sp. y Bufo sp.), 3 órdenes de insectos (Coleoptera, Diptera y 1 grupo no identificado) y 1 género de planta Leucaena spp.

El análisis de $\mathrm{X}^{2}$, con los diferentes tamaños de muestra permitió determinar que el número mínimo de excrementos necesarios para describir la dieta de la nutria en Temascalpetec, México es de $10\left(\mathrm{X}^{2}=16.12\right.$, g.l. $=6$, $\mathrm{p}<0.05)$.

El índice de Levin para la época seca fue 0.03 y para la lluviosa 0 . El índice de Levin para el río Telpintla fue 0 y para el río Grande fue 0.08. Para la comparación entre diversos estudios, se obtuvo el índice de Levin de los trabajos de Spínola y Vaughan (1995), Gallo-Reynoso (1989), Macías-Sánchez y Aranda (1999) y Kasper et al. (2004) (Cuadro 2). El solapamiento de nicho trófico (índice de Pianka) entre estaciones (secas-lluvias) fue de

Cuadro 1. Frecuencia (F), frecuencia de presencia (FP) y porcentaje de aparición (PA) de los grupos-presa encontrados en 157 excrementos de Lontra longicaudis recolectados en 2 ríos de Temascaltepec, Estado de México, entre noviembre de 2004 y junio de 2006

\begin{tabular}{lccc}
\hline & $F$ & $F P(\%)$ & $P A$ \\
Grupos-presa & $(N=157)$ & $N=157$ & $(N 1=171)$ \\
\hline Peces & $\mathbf{1 5 7}$ & $\mathbf{1 0 0}$ & $\mathbf{9 1 . 8 2}$ \\
O. mykiss & 157 & 100 & 92 \\
Anfibios & $\mathbf{5}$ & $\mathbf{3 . 1 8}$ & $\mathbf{2 . 9 2}$ \\
Hyla spp. & 4 & 2,55 & 2,3 \\
Bufo spp. & 1 & 0,63 & 0,6 \\
Invertebrados & $\mathbf{6}$ & $\mathbf{3 . 8 2}$ & $\mathbf{3 . 5}$ \\
Coleóptero & 2 & 1,28 & 1,1 \\
Díptero & 1 & 0,63 & 0,6 \\
& & & \\
Insectos no identificados & 3 & 1,9 & 1,7 \\
Plantas & $\mathbf{3}$ & $\mathbf{1 . 9 1}$ & $\mathbf{1 . 7 6}$ \\
Leucaena sp. & 3 & 1,9 & 1,7 \\
Total & 171 & & 100 \\
\hline
\end{tabular}

Cuadro 2. Valores de nicho trófico estandarizado (B') de $L$. longicaudis obtenidos en varias zonas de estudio

\begin{tabular}{lcc}
\hline Autor & $B^{\prime}$ & $\begin{array}{c}\text { Núm. de presas } \\
\text { (grupos-presa) }\end{array}$ \\
\hline Gallo-Reynoso, (1989) & 0.3 & 32 \\
Spínola y Vaughan, (1995) & Río $1=0.3$ & 15 \\
& Río 2=0.3 & 15 \\
Macías-Sánchez y Aranda, (1999) & 0.5 & 16 \\
Kasper et al., (2004) & Río 1=0.2 & 17 \\
Este estudio & Río 2=0.2 & 14 \\
\hline
\end{tabular}


1. El solapamiento trófico entre ríos fue de 0.99. En ambas temporadas la presa de mayor aparición fue O. mykiss, se obtuvieron 150 muestras para la época seca y 7 para la época húmeda. No se presentó variación significativa en la dieta de la nutria entre temporadas $\left(\mathrm{X}^{2}=0.41\right.$; g.l. $=1$; $\mathrm{p}>$ $0.93)$, aunque si entre ríos $\left(\mathrm{X}^{2}=14.75\right.$, g.l. $\left.=1, \mathrm{p}<0.002\right)$, donde se obtuvieron 87 en el río Telpintla y 70 en el río Grande. En ambos ríos el grupo de mayor aparición fueron los peces, sin embargo en el río Grande se registro más de una presa en la dieta de L. longicaudis. No obstante, no existieron variaciones importantes entre los dos ríos a nivel de grupos-presa, ya que tanto en el río Telpintla como en el río Grande predominó $O$. mykiss.

\section{Discusión}

La dieta de L. longicaudis estuvo compuesta por peces, invertebrados, anfibios y plantas. La trucha arco iris $(O$. mykiss) presentó el porcentaje de aparición más elevado tanto espacial como temporalmente por lo que se considera como la principal presa de L. longicaudis en la zona de estudio. En la zona de estudio existen varios criaderos de trucha; se observó que los peces tienen poca vigilancia, ya que varias truchas se escapan al cauce de los ríos. El río Temascaltepec, pertenece a la faja volcánica transmexicana que para el Estado de México es una zona poco estudiada (Luna et al., 2007), este río vierte hacia el sur, sitio alto donde inicia la cuenca del Balsas que es la menos estudiada en el Estado de México, de ésta se conocen pocas especies de peces (Méndez-Sánchez et al., 2002). Se presume que en la zona de estudio debieron presentarse especies como Girardinichtys multiradiatus y Chirostoma humboltianum, mismos que no han sido recolectados recientemente. En muestreos con equipo de electropesca en uno de los ríos, el único pez que se encontró fue la trucha arco iris, mientras que en el otro río no se encontraron peces.

El tamaño de muestra para la determinación de los hábitos alimentarios de la nutria difiere en cada trabajo, se considera que está directamente relacionado con las condiciones de cada río y el tiempo de recolecta (Pardini, 1998; Macías-Sánchez y Aranda, 1999; Kasper et al., 2004). Estas condiciones hacen referencia a las características que los ríos presenten; como tamaño del cauce y abundancia y diversidad de presas. Los ríos donde se realizaron las recolectas presentaban zonas de rápidos y pozas profundas; sin embargo, en el río Telpintla se observó un efecto negativo sobre las poblaciones de peces así como en la nutria, al establecerse cerca de su cauce un rancho ganadero que vertía desechos orgánicos al río.

El tamaño de muestra necesario para describir la dieta de la nutria en la zona fue de 10. Es la primera vez que se menciona este análisis para esta especie; el valor está en función del tamaño de la muestra total, así como en la diversidad de presas. En este caso, en el $90 \%$ de las muestras se presentó una sola especie; además, solamente están registrados 7 grupos de presas. El tamaño de muestra necesario para describir la dieta de otros carnívoros ya se ha registrado usando el mismo método (Monroy-Vilchis et al., 2009).

Los estudios realizados en otras zonas mencionan que las nutrias son una especie generalista debido a que dentro de su dieta se encuentra incluido un gran número de especies, desde invertebrados (insectos y crustáceos) hasta vertebrados, como peces, anfibios, reptiles, aves y pequeños mamíferos (Gallo-Reynoso, 1989). La conclusión de este estudio únicamente considera la presencia de las especies presa y no así su frecuencia de aparición en los excrementos, lo cual es importante para determinar la condición de generalista o de especialista (Krebs, 1999).

Los resultados del presente estudio, usando el índice de Levin, sugieren que la nutria es una especie con tendencia a especialista en el área de estudio ya que los valores de diversidad trófica de la especie son muy cercanos a cero. Por otro lado, los índices de otros estudios analizados también sugieren que la nutria es de hábitos especialistas ya que los valores del índice de Levin en la mayoría de los casos son menores a 0.5 . Esto probablemente evidencia una falta de profundidad en los estudios de dieta de nutria para poder hacer inferencias con mayor precisión. La nutria es una especie de amplia distribución que presenta tendencia especialista en su dieta en sitios específicos, pero en todo su rango de distribución puede alimentarse de una gran variedad de especies. Es evidente que la dieta de los organismos con amplia distribución cambiará dependiendo del sitio donde se localicen las distintas poblaciones, como sucede en el caso del puma (Monroy-Vilchis et al., 2009).

De igual forma, en relación con las temporadas climáticas, los valores son muy cercanos a cero, lo que fortalece la idea de tendencia especialista de la nutria en la zona. La comparación de los resultados con otros estudios muestra que la alimentación de la nutria presenta similitud en cuanto a los grupos-presa, dado que la base son crustáceos y peces. Sin embargo, al considerar todo el espectro alimentario, éste cambia notablemente. MacíasSánchez y Aranda (1999) mencionan 2 grupos de presas, reptiles y aves, además de los registrados en este estudio; sin embargo, las plantas no aparecen como parte de la dieta,siendo los peces el grupo más importante en ambos estudios. También Pardini (1998) y Kasper et al. (2004) evidencian a los peces como la presa principal de $L$. longicaudis; sólo Spínola y Vaughan (1995), registraron los crustáceos como la presa principal para la nutria. No obstante que en este estudio no se registraron restos de 
crustáceos en los excrementos que fueron analizados, en la zona sí se registraron organismos de este grupo que potencialmente podrían ser utilizados como presas para las nutrias; sin embargo, tal parece que la disponibilidad de peces en los ríos es suficiente como para que éstas no depreden sobre este tipo de organismos. Además, es probable que la biomasa aportada por las truchas sea más elevada que la que le proporcionaría el consumo de crustáceos.

El analisis de los datos obtenidos para el solapamiento trófico nos muestra que existe una completa sobreposición en cuanto a los recursos usados por las nutrias en ambos ríos, indicándonos que las truchas están a lo largo de ambos cauces y que los criaderos son un importante proveedor de estos organismos. En la zona, la variación temporal es marcada en cuanto a las condiciones climáticas; no obstante, los grupos de presa no cambian, ya que $O$. mykiss es la presa principal, tanto en época de sequía como en época lluviosa. Este efecto puede presentarse debido a que la trucha es una especie introducida en la zona y su reproducción está regulada por el hombre; por tanto, su variación en la disponibilidad no depende de ciclos naturales. En otros estudios se observa que las temporadas climáticas marcan claramente una pauta en relación a la alimentación de la nutria, por ejemplo Macías-Sánchez (1998) observa que durante la época lluviosa las nutrias son piscívoras y durante la época seca su dieta se compone principalmente de crustáceos.

En los ríos de Temascaltepec que se han evaluado, donde aún se encuentran presentes las nutrias, la densidad humana es baja; aún así, la presencia de criaderos de trucha ha modificado el comportamiento de las nutrias, dado que éstas prefieren permanecer cerca de las zonas donde se han establecido, lo que sugiere que la presencia humana ha creando un ambiente artificial favorable para las nutrias, haciéndolas dependientes de las condiciones que el manejo de las granjas piscícolas imponen en su entorno, por lo que ahora su permanencia está probablemente relacionada con la estabilidad de las poblaciones de las truchas, sobre todo si se considera que no se obtuvieron capturas de peces distintos a las truchas en los ríos donde se efectuaron muestreos; aunque esta observación se tiene que analizar con mayor detalle para determinar como afectaría a la nutria la remoción de los criaderos de truchas. Tanto el grado de especialización de L. longicaudis por alimentarse de una especie (trucha) y que esta especie sea introducida y criada bajo condiciones controladas sugieren que la estabilidad y permanencia de la nutria en la zona sea de alto riesgo, ya que su conducta alimentaría puede impactar directamente en los intereses económicos de los habitantes locales. Hasta el momento, sólo se tiene el registro de una nutria muerta, en 2005, debido a que fue sorprendida entrando a un criadero a comer truchas. Por otro lado, esto es una evidencia interesante de la flexibilidad en la conducta y la dieta que puede presentar la nutria en sitios modificados por las actividades humanas, específicamente por los criaderos de peces comerciales como la trucha arco iris.

\section{Agradecimientos}

A la Estación Biológica Sierra Nanchititla, Universidad Autónoma del Estado de México por el apoyo otorgado para el presente proyecto. A X.Guerrero por su colaboración en el trabajo de campo. A S. Macías por sus comentarios que fortalecieron el manuscrito. A Juan Pablo Gallo y dos revisores anónimos cuyos comentarios enriquecieron el manuscrito.

\section{Literatura citada}

Aranda, M. 2000. Huellas y otros rastros de los mamíferos grandes y medianos de México. Comisión Nacional para el Conocimiento y Uso de la Biodiversidad-Instituto de Ecología, Xalapa, Veracruz 212 pp.

Borboa Reyes, A. 1999. Temascaltepec, Monografía Municipal. Instituto Cultural Mexiquense. 249 pp.

Braña, F., J. Naves y G. Palomero. 1987. Hábitos alimenticios y configuración de la dieta del oso pardo en la cordillera cantábrica. Coloquio Internacional sobre el oso pardo, 14-15 de noviembre de 1987, Seix (Arilge), Francia. 1-15 p.

Casariego-Madorell, A. A., R. List y G. Ceballos. 2006. Aspectos básicos sobre la ecología de la nutria de río (Lontra longicaudis annectens) para la costa de Oaxaca. Revista Mexicana de Mastozoología 10: 71-74.

Caselli, F. y D. Murray. 2007. Evaluation of potential factors predisposing livestock to predation by jaguars. Journal of Wildlife Management 71: 2379-2386.

Fierros-López, H. E. 2003. Guía para las familias comunes de coleoptera de México. Anónimo, México, D.F. 41 p.

Gallo-Reynoso, J. P. 1987. Reconocimiento del hábitat y alimentación del perro de agua (Lutra longicaudis annectens Major, 1897), en la cuenca del río Nexpa, Guerrero, México. Memorias del Simposio Internacional de Mastozoología Latinoamericana, Cancún, Quintana Roo, junio de 1987. s/p.

Gallo-Reynoso, J. P. 1989. Distribución y estado actual de la nutria o perro de agua (Lutra longicaudis annectens Major, 1897) en la sierra Madre del Sur, México. Tesis, maestría Facultad de Ciencias, Universidad Nacional Autónoma de México, México, D.F. 236 p.

Gallo-Reynoso, J. P. 1996. Distribution of the neotropical river otter (Lontra longicaudis annectens Major, 1897) in the Río Yaqui, Sonora México. IUCN. Otter Specialist Group Bulletin 13:27-31.

Gallo-Reynoso, J. P. 1997. Situación y distribución de la nutria en México, con énfasis en Lontra longicaudis annectens 
Major, 1897. Revista Mexicana de Mastozoología 2:10-32.

Gallo-Reynoso, J. P., N.N. Ramos-Rosas y O. Rangel-Aguilar. 2008. Depredación de aves acuáticas por la nutria neotropical (Lontra longicaudis annectens), en el río Yaqui, Sonora, México. Revista Mexicana de Biodiversidad 79:275-279.

Gittleman, J. L. 1989. Carnivore behavior, ecology, and evolution. Chapman \& Hall, London. 620 p.

Guerrero F. J. J. 2007. Evaluación del hábitat de la nutria (Lontra longicaudis) en tres ríos de Temascaltepec, Estado de México. Tesis, Facultad de Ciencias, Universidad Autónoma del Estado de México. 37 p.

Guerrero, S., M. H. Badii., S. S. Zalapa y A. E. Flores. 2002. Dieta y nicho de alimentación del coyote, zorra gris, mapache, y jaguarundi en un bosque tropical caducifolio de la costa del sur del estado de Jalisco. México. Acta Zoológica Mexicana (ns) 86:119-137

INEGI (Instituto Nacional de Estadística, Geografía e Informática. 2008. México, información geográfica, datos generales. http://www.inegi.gob.mx: 16. IV.2008.

Kasper, B. C., M. J. Feldens., J. Salvi y H. C. Z. Grillo. 2004. Estudo preliminar sobre ecologia de Lontra longicaudis (Olfers) (Carnivora, Mustelidae) no vale do Taquari, sui do Brasil. Revista Brasileira de Zoología 21:65-72.

Krebs, C. J. 1999. Ecological methodology, segunda edición, Addison Wesley, Longman, Harlow, Essex. 620 p.

Kruuk, H. 1995. Wild otters. Predation and populations. Oxford University Press. 290 p.

Larivière, S. 1999. Lontra longicaudis. Mammalian Species 609:1-5.

Lodé, T. 1993. The decline of otter Lutra lutra populations in the region of the Pays de Loire, western France. Biological Conservation 65:9-13.

Luna, I., J. J. Morrone y D. Espinosa (eds.). 2007. Biodiversidad de la faja volcánica transmexicana. Universidad Nacional Autónoma de México, México. 514 p.

Macías-Sánchez, S. 1998. Análisis de los hábitos alimentarios de la nutria neotropical Lontra longicaudis (Olfers, 1818) en un sector del río Los Pescados, municipio de Jalcomulco, Veracruz. Tesis, Facultad de Biología, Universidad Veracruzana, Xalapa, Veracruz. 50 p.

Macías-Sánchez, S. y M. Aranda. 1999. Análisis de la alimentación de la nutria Lontra longicaudis (Mammalia: Carnivora) en un sector del río Los Pescados, Veracruz, México. Acta Zoológica Mexicana (ns) 76:49-57.

Méndez-Sánchez, F., E. Soto, J. P. Maya y M. A. Hernández. 2002. Ictiofauna del Estado de México. Ciencia ergo sum 9:87-90.
Michalski, F., R. L. P. Boulhosa, A. Faria y C. A. Pérez. 2006. Human-wild conflicts in a fragmented Amazonian forest landscape: determinants of large felids depredation on livestock. Animal Conservation 9:179-188.

Monroy-Vilchis, O. y R. Rubio-Rodríguez. 2003. Guía de mamíferos terrestres del Estado de México, a través del pelo de guardia. Universidad Autónoma del Estado de México, Toluca, $115 \mathrm{p}$.

Monroy-Vilchis, O., Y. Gómez, M. Janczur y V. Urios. 2009. Food niche of Puma concolor in central Mexico. Wildlife Biology 15:97-105.

Pardini, R. 1998. Feeding ecology of the neotropical river otter Lontra longicaudis in an Atlantic forest stream, souhteastern Brazil. Journal of Zoology 254:385-391

Ramón, J. 2000. Hábitos alimentarios de la nutria o perro de agua (Lutra longicaudis Major) en una fracción del río San Cipriano del municipio de Nacajuca, Tabasco, México. Tesis, Universidad Juárez Autónoma de Tabasco. Villahermosa. $37 \mathrm{p}$.

Ruiz-Olmo, J. y M. Delibes. 1998. La nutria en España ante el horizonte del año 2000. SECEM (Sociedad Española para la Conservación y Estudio de los Mamíferos) Grupo Nutria, Barcelona-Sevilla-Málaga. 288 p.

SEMARNAT (Secretaría del Medio Ambiente y Recursos Naturales). 2002. Norma Oficial Mexicana. NOM-Ecol-0592001, Protección ambiental-Especies nativas de México de flora y fauna silvestres-Categorías de riesgo y especificaciones para su inclusión, exclusión o cambio-Lista de especies en riesgo. Diario Oficial de la Federación (segunda sección), 6 de marzo, México, D. F.

Servín, J. y C. Huxley. 1991. La dieta del coyote en un bosque de encino-pino de la sierra Madre occidental de Durango, México. Acta Zoológica Mexicana (ns) 44:1:26

Soler, A. 2002. Nutrias por todo México. Biodiversitas 7:13-14.

Sierra-Huelsz, J. A. y J. A. Vargas-Contreras. 2002. Registros notables de Lontra longicaudis annectens (Carnivora: Mustelidae) en el río Amacuzac en Morelos y Guerrero. Revista Mexicana de Mastozoología 6:129-135.

Spínola, R. M. y C. Vaughan. 1995. Dieta de la nutria neotropical (Lutra longicaudis) en la estación biológica La Selva, Costa Rica. Vida Silvestre Neotropical 4:125-132.

UNEP-WCMC (United Nations Environment Programme-World Conservation Monitoring Centre). 2007. Species database. CITES-listed species. http://www.cites.org; consulta 16.09.2008

Zar, J. H. 1999. Biostatistical analysis, fourth edition. Prentice Hall, New Jersey. 663 p. 